\title{
Prevalence and predictors of undernutrition among children under two years in Narmada District, Gujarat State, Western India: a community-based cross-sectional study
}

\section{Authors}

Ritu Rana*, Ankita Sharma, Radhika Nampurkar and Divya Nair H

Affiliation

Indian Institute of Public Health Gandhinagar, Gandhinagar, Gujarat, 382042, India

*Correspondence

rrana@iiphg.org

https://orcid.org/0000-0001-6071-1750 


\section{ABSTRACT}

Background: Globally, addressing undernutrition has been recognised as a development priority. In India, undernutrition continues to be the topmost risk factor for morbidity and associated mortality. The Government of India, though the National Nutrition Strategy, aims to reduce child mortality by preventing and reducing undernutrition among children. The aim of this study was to estimate prevalence and predictors of undernutrition among children under two years in Narmada district of Gujarat state, India.

Methods: A community-based cross-sectional survey was completed between July and August 2019. A probability sample of 400 children under two years of age were surveyed. A structured tool was used to collect data on exposures- socioeconomic conditions, dietary diversity, maternal care, infant feeding practices, household food insecurity, and child morbidity. The WHO child growth standards (2006) were used to measure the outcome (wasting, stunting and underweight). We used multivariate logistic regression analysis to identify the predictors of undernutrition.

Results: The prevalence of wasting, stunting, and underweight were 32.2\% (95\% CI: 27.037.9), 34.5\% (95\% CI: 29.5-39.8), and 39.7\% (95\% CI: 33.9-45.8), respectively. Household income $>10000$ Indian Rupees-INR (OR: 0.61, 95\% CI: 0.4, 0.90), maternal stature $\geq 145 \mathrm{~cm}$ (OR: 0.43, 95\% CI: 0.21, 0.86) and child age 0-6 months (OR: 1.61, 95\% CI: 1.00-2.56) were significantly associated with wasting. Household income (OR: 0.50, 95\% CI: 0.26, 0.97), livestock ownership (OR 2.36: 95\% CI: 1.25, 4.44), maternal body mass index $\geq 18.5$ (OR: 0.63, 95\% CI: 0.42, 0.95), and child age 7-23 months (OR: 5.89, 95\% CI: 3.03, 11.45) were significantly associated with stunting. Maternal stature $\geq 145 \mathrm{~cm}$ (OR: $0.46,95 \%$ CI: 0.23 , 0.95) was significantly associated with underweight.

Conclusions: In Narmada, the prevalence of undernutrition among children under two years is high. To improve the situation, targeting the nutritional status of adolescent girls and mothers, strengthening already existing programs, and education on hygiene should be considered.

Keywords: Malnutrition, wasting, stunting, underweight, prevalence, children 


\section{INTRODUCTION}

Maternal and child undernutrition are among the world's most serious economic, health and human development challenges. Annually, undernutrition contributes to an estimated 3.1 million (45\%) child deaths worldwide [1]. Maternal and child undernutrition have adverse consequences for pregnancy outcomes, child morbidity and mortality, children's mental and motor development, and chronic diseases in later life [2]. The impact of undernutrition is not only limited to the health-related outcomes only, but can also affect educational performance, economic productivity, and human wellbeing [2].

Addressing the burden of undernutrition has been recognised as a global priority- in 2012 the World Health Organization (WHO) specified six global nutrition targets to be achieved by 2025 and in 2016 the United Nations (UN) Decade of Action on Nutrition (2016-25) was launched [3, 4]. Yet, globally in 2018, an estimated 149 million (22\%) children under five years were stunted and 49 million (7.3\%) were wasted [5]. Although there has been some progress in achieving the global nutrition targets, many high-burden countries are off-course [6].

As one of the high burden countries, 38.4\% young children in India are stunted, $35.7 \%$ underweight and $21.0 \%$ wasted children [7]. Undernutrition continues to be the topmost risk factor for morbidity and associated mortality in India- accounting for more than two-third of under-five deaths [8]. In line with the Global Development Goals, the Government of India, in 2017, developed a National Nutrition Strategy (NNS) with the vision of a "malnutrition free India” by 2022 [9]. This strategy aims to reduce child mortality by preventing and reducing undernutrition among children by three percentage points per annum. Subsequently, in 2018, the National Nutrition Mission, also known as the Prime Minister's Overarching Scheme for Holistic Nourishment (POSHAN Abhiyan) was launched [10]. It is considered to be a "People's Movement" and mainly focuses on strengthening the already existing programs through convergence, community engagement, partnerships and use of information technology.

To accelerate nutrition actions under POSHAN Abhiyan, high burden districts across the states have been identified by the NNS. Ever since these districts were identified, many developmental partners and non-governmental organisations have collaborated with the Department of Health and Family Welfare (DHFW) and the Department of Women and Child Development (DWCD) to support nutrition actions. A large nutrition project is one of such initiatives being implemented in Narmada district- one of the high burden districts in Gujarat state.

This paper presents the results of baseline survey for a large nutrition project that aims to prevent wasting among children under two years of age in Narmada district, Gujarat state, India.

\section{METHODS \\ Study setting}

The study was conducted in Narmada district, Gujarat State, western India. The district has a total population of 590,297 (90\% rural and 82\% tribal). Overall, there are 558 inhabited villages and 5 towns. The district has a literacy rate of $72 \%$ and the economy is basically dependent on agricultural activities (85\%). 


\section{Study design and sample size}

A cross-sectional design was used to collect data during July and August 2019. This survey was designed to provide baseline information on nutritional status of children under two years in Narmada district. Sample size was determined using a comparison of repeated survey approach to assess the impact of an intervention over time. The calculation was based on the following assumptions: $36 \%$ prevalence of wasting, $5 \%$ level of significance, $80 \%$ power, $5 \%$ non-response rate and a design effect of 2 . We estimated that a total sample size of 400 was sufficient to detect a minimum change of 15 percentage points in proportion undernourished.

\section{Sampling technique and procedures}

A two-stage cluster sampling technique was used to first select the villages (clusters) and then the households. In the first stage, 40 villages were selected using a probability proportional to size technique. In the second stage, a list of households with infants under two years old was collected from respective village heads. Ten households per village were randomly selected for interview. We used computer generated random numbers.

\section{Data collection procedure and quality assurance}

A structured questionnaire was used to collect data through face-to-face interviews from mothers of sample children. The information collected included demographic and socioeconomic characteristics; dietary diversity; infant and young child feeding practices (IYCF); maternal antenatal-delivery-postnatal care; household food insecurity (HFI); morbidity; and anthropometric measurements. The questionnaire was developed in English and then translated into the local language (Gujarati) by the research team. This was field tested and revised accordingly.

Dietary diversity was measured for both the mothers and children using FAO/FANTA and WHO guidelines, respectively $[11,12]$. We used a 24-hour dietary recall method to calculate the number of food groups consumed. For mothers, the foods were classified into ten groups: i) grains, white roots, and tubers, and plantains; ii) pulses (beans, peas, and lentils); iii) nuts and seeds; iv) dairy; v) meat, poultry, and fish; vi) eggs; vii) dark green leafy vegetables; viii) other vitamin A-rich fruits and vegetables; ix) other vegetables; and x) other fruits. For children the foods were classified into seven groups: i) grains, roots and tubers; ii) legumes and nuts; iii) dairy products (milk, yogurt, cheese); iv) flesh foods (meat, fish, poultry and liver/organ meats); v) eggs; vi) vitamin-A rich fruits and vegetables; and vii) other fruits and vegetables. Women's diet were considered diverse if they consumed food from at least five food groups out of ten, while children's diet was considered diverse if they consumed food from at least four food groups out of seven. Based on this, a minimum dietary diversity (MDD) score was calculated for each.

IYCF indicators were constructed as per WHO guidelines [12]. We measured four indicators: early initiation of breastfeeding, duration of exclusive breastfeeding, timely introduction of complementary foods, and minimum dietary diversity.

HFI was measured using Food and Nutrition Technical Assistant (FANTA) project guidelines [13]. We asked nine questions to mothers related to the household's experience of food insecurity in the last month preceding the survey. These questions had three major domains of HFI: i) anxiety and uncertainty about access (1-question), ii) insufficient quality (3questions), and iii) insufficient quantity (5-questions). Each item starts with an occurrence question that identifies if the condition has been experienced in the household. An affirmative answer is then followed by a frequency-of-occurrence question to determine if the condition 
happened rarely (once or twice), sometimes (3-10 times), or often ( $>10$ times) during the last month. The responses were coded as- $1=$ rarely, $2=$ sometimes, or $3=$ often. From these questions, we formed the Household Food Insecurity Access Prevalence (HFIAP) indicator. Using this, we categorised household security into two levels of HFI: food secure and food insecure.

Anthropometry included weight (mother-child) and height/length (mother-child) measurements using protocols described in FANTA guidelines [14]. Weight for the mother was measured to the nearest $0.1 \mathrm{~kg}$ (Seca 874 electronic scale) and for children to the nearest $0.1 \mathrm{~kg}$ (Seca 364 infant electronic scale). Similarly, height (Seca 213 stadiometer) and length (Seca 210 measuring mat) for mother and child were measured to the nearest $1 \mathrm{~cm}$.

The data collection team included two research assistants (RA) and eight field investigators (FI). The RAs had formal training in public health nutrition, including a focus on anthropometry. The FIs were graduates from local areas with some fieldwork experience. The questionnaire was administered by FIs, while anthropometry was performed by the RAs. Two separate training sessions were conducted, one for RAs and other for FIs. RAs were trained at the Indian Institute of Public Health Gandhinagar (IIPHG) by the principal investigator. This included the conceptual framework for malnutrition, survey design, sampling techniques and data collection. The FIs were trained at Rajpipla village in Narmada (a field station) by the RAs. Their training mainly focused on the survey questionnaire.

There were two levels of supervision, ensuring the quality of the data collection: RAs (field supervisors) and a remote supervisor (principal investigator). The field supervisors were responsible for checking anthropometric measurements and observing interviewers throughout the survey. Collected data were entered into Excel the same day by RAs and were shared with the principal investigator for checking. Any inaccuracies found were resolved the next day.

\section{Data processing and analysis}

The raw data were cleaned, coded and analysed (adjusted for cluster sampling) using STATA version 15. Three anthropometric indicators were generated: weight-for-height/length (WHZ), height/length-for-age (HAZ), and weight-for-age (WAZ), using the WHO child growth standards [15-17]. These were used to generate outcome indicators--wasted, stunted or underweight as WHZ, HAZ, and WAZ scores <-2 standard deviations (SD), respectively.

Firstly, we used the univariate analysis to examine the distributions of variables. This was used to understand the study population characteristics. The results are presented as percentages for categorical variables and means with SD for continuous variables. Secondly, we calculated the prevalence of anthropometric deficits (wasted, stunted, and underweight) among children. Thirdly, we performed a bivariate analysis to assess associations between exposure (household, maternal and child related) and outcome variables (wasting, stunting and underweight). Those found to be significantly associated $(\mathrm{p}<0.05)$ were then included in the multivariate analysis. Finally, logistic regression (multivariate) analysis was performed to investigate the net effect of each exposure on wasting, stunting and underweight, adjusting for all other variables in the model. These results are presented as adjusted odds ratios (OR) with $95 \%$ confidence intervals.

\section{Ethics approval and consent to participate}

Ethics approval and clearance was obtained from the Institution Ethics Committee of Indian Institute of Public Health Gandhinagar (IIPHG). Study participants (mothers) were informed 
about the research objectives, methods, and techniques in detail and written informed consent was taken.

\section{RESULTS}

\section{Characteristics of study population}

A total of 400 mother-child pairs participated in the study. Characteristics of household, mothers and their children are presented in Table 1. A majority of the households were Hindus and belonged to the scheduled-castes/scheduled-tribes (SC/ST) category. Regarding socio-economic conditions, $61.5 \%$ had a monthly family income $\leq 10,000$ INR (140 USD) and nearly three-quarters (73.5\%) owned livestock. Two-thirds of households were living in kutcha (made of mud or thatch) and the rest in pucca (made of stones, asbestos sheets, burnt bricks, cement bricks or concrete) houses and around half did not have their own toilets. Most (86.2\%) of the households reported being food secure.

Table 1: Socio-economic and demographic characteristics of children under 2 years and their mothers, in Narmada district, Gujarat state, Western India

\begin{tabular}{|c|c|c|c|}
\hline Characteristics & Category & Frequency & Percent (\%) \\
\hline \multirow[t]{3}{*}{ Religion } & Hindu & 377 & 94.2 \\
\hline & Muslim & 16 & 4.0 \\
\hline & Christian & 7 & 1.8 \\
\hline \multirow[t]{3}{*}{ Caste } & General & 9 & 2.2 \\
\hline & $\mathrm{SC} / \mathrm{ST}$ & 376 & 94.0 \\
\hline & OBC & 15 & 3.8 \\
\hline \multirow[t]{2}{*}{ Monthly family income (₹) } & $\leq 10000$ & 246 & 61.5 \\
\hline & $>10000$ & 154 & 38.5 \\
\hline \multirow[t]{2}{*}{ Family type } & Joint & 105 & 26.2 \\
\hline & Nuclear & 295 & 73.8 \\
\hline \multirow{2}{*}{ House type } & Kutcha & 265 & 66.2 \\
\hline & Pucca & 135 & 33.8 \\
\hline \multirow[t]{2}{*}{ Land ownership } & Yes & 332 & 83.0 \\
\hline & No & 68 & 17.0 \\
\hline \multirow[t]{2}{*}{ Livestock ownership } & None & 106 & 26.5 \\
\hline & $\geq 1$ & 294 & 73.5 \\
\hline \multirow[t]{2}{*}{ Toilet availability } & Yes & 203 & 50.8 \\
\hline & No & 197 & 49.2 \\
\hline \multirow[t]{2}{*}{ Household food insecurity } & Secure & 345 & 86.2 \\
\hline & Insecure & 55 & 13.8 \\
\hline
\end{tabular}

Notes: SC- scheduled-castes; ST- scheduled-tribes; OBC- other so-called backward castes

Regarding maternal characteristics, the mean age was $24.8 \pm 3.75$ years and most (83.5\%) were literate (Table 2 ). A small proportion (13.2\%) reported early marriage ( $<18$ years). Maternal antenatal, delivery and postnatal care were found to be satisfactory. The mean body mass index (BMI) was 19.4 \pm 3.09 , with $44 \%$ mothers being underweight and $7.5 \%$ having short stature $(<145 \mathrm{~cm})$. The mean dietary diversity score for mothers was $4.3 \pm 0.99$ and $43.2 \%$ did not meet the minimum dietary diversity. Regarding infant and young child feeding practices, three indicators were satisfactory (exclusive breastfeeding- 97\%, early initiation- $80.5 \%$, and minimum dietary diversity- $71.4 \%$ ), while the timely introduction of complementary feeding was low (55.8\%). The mean age of children was $10.6 \pm 6.31$ months. The male-female ratio was nearly equal. $16.8 \%$ of children were reported to have had diarrhoea in last two weeks. 
Table 2: Maternal characteristics and IYCF practices

\begin{tabular}{|c|c|c|c|}
\hline Characteristics & Category & Frequency & Percent (\%) \\
\hline Maternal age, years (Mean \pm SD) & & $24.8 \pm 3.75$ & \\
\hline \multirow[t]{2}{*}{ Maternal education } & Literate & 334 & 83.5 \\
\hline & Illiterate & 66 & 16.5 \\
\hline \multirow[t]{2}{*}{ Age at marriage, years } & $<18$ & 53 & 13.2 \\
\hline & $\geq 18$ & 347 & 86.8 \\
\hline \multirow[t]{2}{*}{ Parity } & $1-3$ & 370 & 92.5 \\
\hline & $\geq 4$ & 30 & 7.5 \\
\hline \multirow[t]{2}{*}{ Maternal height, $\mathrm{cm}$} & $<145$ & 45 & 11.2 \\
\hline & $\geq 145$ & 355 & 88.8 \\
\hline Maternal BMI, kg/m² (Mean \pm SD) & & $19.4 \pm 3.09$ & \\
\hline \multirow[t]{2}{*}{ Maternal nutrition status } & $\mathrm{BMI}<18.5$ & 176 & 44 \\
\hline & $\mathrm{BMI} \geq 18.5$ & 224 & 56 \\
\hline Maternal dietary diversity (Mean \pm SD) & & $4.3 \pm 0.99$ & \\
\hline \multirow[t]{2}{*}{ MDD-M } & Yes & 227 & 56.8 \\
\hline & No & 173 & 43.2 \\
\hline \multirow{2}{*}{ Antenatal visits } & $\geq 4$ times & 297 & 74.2 \\
\hline & $<4$ times & 103 & 25.8 \\
\hline \multirow[t]{2}{*}{ Place of delivery } & Home & 34 & 8.5 \\
\hline & Institution & 366 & 91.5 \\
\hline \multirow[t]{2}{*}{ Delivery type } & Normal & 366 & 91.5 \\
\hline & Caesarean & 34 & 8.5 \\
\hline \multirow[t]{2}{*}{ Assistance during delivery } & Yes & 348 & 87.0 \\
\hline & No & 52 & 13.0 \\
\hline \multirow{2}{*}{ Received postnatal care } & Yes & 366 & 91.5 \\
\hline & No & 34 & 8.5 \\
\hline \multirow{2}{*}{ Early initiation of breastfeeding } & Yes & 323 & 80.8 \\
\hline & No & 77 & 19.2 \\
\hline \multirow[t]{2}{*}{ Exclusive breastfeeding (0-5 months) } & Yes & 98 & 97.0 \\
\hline & No & 3 & 3.0 \\
\hline \multirow{2}{*}{$\begin{array}{l}\text { Timely introduction of complementary feeding (6- } \\
8 \text { months) }\end{array}$} & Yes & 29 & 55.8 \\
\hline & No & 23 & 44.2 \\
\hline Child dietary diversity (Mean \pm SD) (7-23 months) & & $2.5 \pm 1.39$ & \\
\hline \multirow[t]{2}{*}{ MDD-C } & Yes & 205 & 71.4 \\
\hline & No & 82 & 28.6 \\
\hline Child age, months (Mean \pm SD) & & $10.6 \pm 6.31$ & \\
\hline \multirow[t]{2}{*}{ Child age group, months } & $0-6$ & 113 & 28.2 \\
\hline & $7-23$ & 287 & 71.8 \\
\hline \multirow[t]{2}{*}{ Child gender } & Male & 206 & 51.5 \\
\hline & Female & 194 & 48.5 \\
\hline \multirow[t]{2}{*}{ Diarrhoea (last two weeks) } & Yes & 67 & 16.8 \\
\hline & No & 333 & 83.2 \\
\hline
\end{tabular}

Note: MDD-M- minimum dietary diversity for mothers; MDD-C- minimum dietary diversity for child 


\section{Prevalence of undernutrition}

The prevalence of wasting, stunting and underweight were 32.2\% (95\% CI: 27.0-37.9), 34.5\% (95\% CI: 29.5-39.8), and 39.7\% (95\% CI: 33.9-45.8), respectively (Table 3). The mean weight-for-height (WHZ), height-for-age (HAZ), and weight-for-age (WAZ) z scores were $-1.37 \pm 0.09,-1.37 \pm 0.10$, and $-1.73 \pm 0.07$, respectively.

Table 3: Prevalence of undernutrition among children under 2 years in Narmada district, Gujarat state, Western India

\begin{tabular}{|c|c|c|}
\hline Indicator & Category & Percentage (95\% CI) \\
\hline \multirow[t]{2}{*}{ Wasted } & $<-2 S D$ & $32.2(27.0-37.9)$ \\
\hline & $<-3 S D$ & $10.5(7.0-15.3)$ \\
\hline \multirow[t]{2}{*}{ Stunted } & $<-2 S D$ & $34.5(29.5-39.8)$ \\
\hline & $<-3 S D$ & $14.7(10.2-17.0)$ \\
\hline \multirow[t]{2}{*}{ Underweight } & $<-2 S D$ & 39.7 (33.9-45.8) \\
\hline & $<-3 S D$ & $13.2(10.2-17.0)$ \\
\hline
\end{tabular}

Table 5 presents the results of adjusted analysis, which identified monthly family income, livestock ownership, maternal height, maternal nutritional status and child age as factors associated with undernutrition.

Monthly family income and child age were associated with both wasting and stunting (though in the latter case, in opposite directions). As shown in Table 5, having a higher income reduced the risk of malnutrition by nearly half. Among older children, the risk of stunting was higher, whereas the risk of wasting was lower.

Livestock ownership and maternal nutritional status were associated with only stunting. Relative to households with no livestock, households with one or more livestock had 2.36 (95\% CI: 1.25-4.44, strong evidence) times odds of having a stunted child. Relative to mothers with BMI $<18.5 \mathrm{~kg} / \mathrm{m}^{2}$, mothers with BMI $\geq 18.5 \mathrm{~kg} / \mathrm{m}^{2}$ had 0.63 (95\% CI: 0.42-0.95, good evidence) times odds of having a stunted child.

Maternal height was associated with both wasting and underweight. Relative to mothers with short stature, mothers with height $\geq 145 \mathrm{~cm}$ had 0.43 (95\% CI: $0.21-0.86$, good evidence) times the odds of having a wasted child and 0.46 (95\% CI: 0.23-0.95, good evidence) times odds of having an underweight child. 


\section{Factors associated with undernutrition and strength of association}

Bivariate analyses identified thirteen factors associated with undernutrition (Table 4). These factors were included in the multivariate analysis.

Table 4: Bivariate analysis showing associations of household, maternal and child characteristics with undernutrition outcomes among children under 2 years in Narmada district, Gujarat state, Western India

\begin{tabular}{|c|c|c|c|c|c|c|c|c|c|}
\hline \multirow{2}{*}{ Variables } & \multicolumn{3}{|l|}{ Wasted } & \multicolumn{3}{|l|}{ Stunted } & \multicolumn{3}{|l|}{ Underweight } \\
\hline & $\begin{array}{l}\text { No } \\
\text { n (\%) }\end{array}$ & $\begin{array}{l}\text { Yes } \\
\text { n (\%) }\end{array}$ & $\begin{array}{l}\text { p- } \\
\text { value }\end{array}$ & $\begin{array}{l}\text { No } \\
\text { n (\%) }\end{array}$ & $\begin{array}{l}\text { Yes } \\
\text { n (\%) }\end{array}$ & $\begin{array}{l}\text { p- } \\
\text { value }\end{array}$ & $\begin{array}{l}\text { No } \\
\text { n (\%) }\end{array}$ & $\begin{array}{l}\text { Yes } \\
\text { n (\%) }\end{array}$ & $\begin{array}{l}\text { p- } \\
\text { value }\end{array}$ \\
\hline \multicolumn{10}{|l|}{ Religion } \\
\hline Hindu & $253(67.1 \%)$ & 124 (32.9\%) & 0.214 & $244(64.7 \%)$ & 133 (35.3\%) & 0.038 & $226(59.9 \%)$ & $151(40.1 \%)$ & 0.001 \\
\hline Muslim & $14(87.5 \%)$ & $2(12.5 \%)$ & & $14(87.5 \%)$ & $2(12.5 \%)$ & & $13(81.2 \%)$ & $3(18.8 \%)$ & \\
\hline Christian & $4(57.1 \%)$ & $3(42.9 \%)$ & & $4(57.1 \%)$ & $3(42.9 \%)$ & & $2(28.6 \%)$ & $5(71.4 \%)$ & \\
\hline \multicolumn{10}{|l|}{ Caste } \\
\hline General & 8 (88.9\%) & $1(11.1 \%)$ & 0.103 & 7 (77.8\%) & $2(22.2 \%)$ & 0.009 & $7(77.8 \%)$ & $2(22.2 \%)$ & 0.005 \\
\hline $\mathrm{SC} / \mathrm{ST}$ & $250(66.5 \%)$ & $126(33.5 \%)$ & & $241(64.1 \%)$ & 135 (35.9\%) & & $220(58.5 \%)$ & $156(41.5 \%)$ & \\
\hline OBC & $13(86.7 \%)$ & $2(13.3 \%)$ & & $14(93.3 \%)$ & $1(6.7 \%)$ & & $14(93.3 \%)$ & $1(6.7 \%)$ & \\
\hline \multicolumn{10}{|c|}{ Monthly family income (₹) } \\
\hline$\leq 10000$ & $156(63.4 \%)$ & $90(36.6 \%)$ & 0.022 & $150(61.0 \%)$ & $96(39.0 \%)$ & 0.042 & 140 (56.9\%) & $106(43.1 \%)$ & 0.103 \\
\hline$>10000$ & $115(74.7 \%)$ & $39(25.3 \%)$ & & $112(72.7 \%)$ & $42(27.3 \%)$ & & $101(65.6 \%)$ & $53(34.4 \%)$ & \\
\hline \multicolumn{10}{|l|}{ House type } \\
\hline Kutcha & $170(64.1 \%)$ & 95 (35.9\%) & 0.043 & $173(65.3 \%)$ & 92 (34.7\%) & 0.914 & $153(57.7 \%)$ & $112(42.3 \%)$ & 0.171 \\
\hline Pucca & $101(74.8 \%)$ & $34(25.2 \%)$ & & $89(65.9 \%)$ & $46(34.1 \%)$ & & $88(65.2 \%)$ & 47 (34.8\%) & \\
\hline \multicolumn{10}{|c|}{ Livestock ownership } \\
\hline None & 76 (71.7\%) & 30 (28.3\%) & 0.279 & $81(76.4 \%)$ & $25(23.6 \%)$ & 0.020 & $69(65.1 \%)$ & 37 (34.9\%) & 0.233 \\
\hline 1 or $>$ & $195(66.3 \%)$ & 99 (33.67\%) & & $181(61.6 \%)$ & $113(38.4 \%)$ & & $172(58.5 \%)$ & $122(41.5 \%)$ & \\
\hline \multicolumn{10}{|c|}{ Toilet availability } \\
\hline Yes & $144(70.9 \%)$ & $59(29.1 \%)$ & 0.234 & $139(68.5 \%)$ & $64(31.5 \%)$ & 0.215 & $133(65.5 \%)$ & $70(34.5 \%)$ & 0.018 \\
\hline No & $127(64.5 \%)$ & $70(35.5 \%)$ & & $123(62.4 \%)$ & $74(37.6 \%)$ & & $108(54.8 \%)$ & $89(45.2 \%)$ & \\
\hline \multicolumn{10}{|c|}{ Age at marriage, years } \\
\hline$<18$ & $34(64.1 \%)$ & 19 (35.9\%) & 0.653 & $27(50.9 \%)$ & 26 (49.1\%) & 0.027 & $28(52.8 \%)$ & $25(47.2 \%)$ & 0.351 \\
\hline$\geq 18$ & 237 (68.3\%) & $110(31.7 \%)$ & & $235(67.7 \%)$ & $112(32.3 \%)$ & & $213(61.4 \%)$ & $134(38.6 \%)$ & \\
\hline \multicolumn{10}{|c|}{ Maternal height, cm } \\
\hline$<145$ & $21(46.7 \%)$ & $24(53.3 \%)$ & 0.006 & $28(62.2 \%)$ & $17(37.8 \%)$ & 0.625 & $19(42.2 \%)$ & 26 (57.8\%) & 0.023 \\
\hline
\end{tabular}




\begin{tabular}{|c|c|c|c|c|c|c|c|c|c|}
\hline \multirow[t]{2}{*}{ Variables } & \multicolumn{3}{|l|}{ Wasted } & \multicolumn{3}{|l|}{ Stunted } & \multicolumn{3}{|l|}{ Underweight } \\
\hline & $\begin{array}{l}\text { No } \\
\text { n (\%) }\end{array}$ & $\begin{array}{l}\text { Yes } \\
\text { n (\%) }\end{array}$ & $\begin{array}{l}\text { p- } \\
\text { value }\end{array}$ & $\begin{array}{l}\text { No } \\
\text { n (\%) }\end{array}$ & $\begin{array}{l}\text { Yes } \\
\text { n (\%) }\end{array}$ & $\begin{array}{l}\text { p- } \\
\text { value }\end{array}$ & $\begin{array}{l}\text { No } \\
\text { n (\%) }\end{array}$ & $\begin{array}{l}\text { Yes } \\
\text { n (\%) }\end{array}$ & $\begin{array}{l}\text { p- } \\
\text { value }\end{array}$ \\
\hline$\geq 145$ & $250(70.4 \%)$ & $105(29.6 \%)$ & & $234(65.9 \%)$ & $121(34.1 \%)$ & & $222(62.5 \%)$ & $133(37.5 \%)$ & \\
\hline \multicolumn{10}{|c|}{ Maternal nutrition status } \\
\hline $\mathrm{BMI}<18.5$ & $114(64.8 \%)$ & $62(35.2 \%)$ & 0.329 & $105(59.7 \%)$ & $71(40.3 \%)$ & 0.011 & $99(56.2 \%)$ & $77(43.8 \%)$ & 0.143 \\
\hline $\mathrm{BMI} \geq 18.5$ & $157(70.1 \%)$ & 67 (29.9\%) & & $157(70.1 \%)$ & $67(29.9 \%)$ & & $142(63.4 \%)$ & $82(36.6 \%)$ & \\
\hline \multicolumn{10}{|c|}{ Place of delivery } \\
\hline Home & 19 (55.9\%) & 15 (44.1\%) & 0.189 & $20(58.8 \%)$ & $14(41.2 \%)$ & 0.349 & $13(38.2 \%)$ & $21(61.8 \%)$ & 0.024 \\
\hline Institution & $252(68.8 \%)$ & $114(31.2 \%)$ & & $242(66.1 \%)$ & 124 (33.9\%) & & 228 (62.3\%) & 138 (37.7\%) & \\
\hline \multicolumn{10}{|c|}{ Child age, months } \\
\hline $0-6$ & $68(60.2 \%)$ & $45(39.8 \%)$ & 0.037 & $98(86.7 \%)$ & $15(13.3 \%)$ & 0.000 & $76(67.3 \%)$ & $37(32.7 \%)$ & 0.125 \\
\hline $7-23$ & 203 (70.7\%) & $84(29.3 \%)$ & & 164 (57.1\%) & $123(42.9 \%)$ & & 165 (57.5\%) & 122 (42.5\%) & \\
\hline \multicolumn{10}{|l|}{ Child gender } \\
\hline Male & $131(63.6 \%)$ & 75 (36.4\%) & 0.047 & $132(64.1 \%)$ & 74 (35.9\%) & 0.424 & $119(57.8 \%)$ & 87 (42.2\%) & 0.329 \\
\hline Female & 140 (72.2\%) & $54(27.8 \%)$ & & $130(67.0 \%)$ & $64(33.0 \%)$ & & $122(62.9 \%)$ & 72 (37.1\%) & \\
\hline \multicolumn{10}{|c|}{ Diarrhoea (last 2 weeks) } \\
\hline Yes & $40(59.7 \%)$ & 27 (40.3\%) & 0.086 & 39 (58.2\%) & $28(41.8 \%)$ & 0.167 & $32(47.8 \%)$ & 35 (52.2\%) & 0.028 \\
\hline No & 231 (69.4\%) & $102(30.6 \%)$ & & $223(67.0 \%)$ & $110(33.0 \%)$ & & 209 (62.8\%) & $124(37.2 \%)$ & \\
\hline
\end{tabular}

Table 5: Multivariate logistic regression analysis showing factors associated with undernutrition among children under 2 years in Narmada district, Gujarat state, Western India

\begin{tabular}{|c|c|c|c|c|c|c|}
\hline \multirow[t]{2}{*}{ Variables } & \multicolumn{2}{|l|}{ Wasting } & \multicolumn{2}{|l|}{ Stunting } & \multicolumn{2}{|l|}{ Underweight } \\
\hline & OR(95\%CI) & p-value & OR(95\%CI) & p-value & OR(95\% CI) & p-value \\
\hline \multicolumn{7}{|l|}{ Religion } \\
\hline Hindu & 1 & & 1 & & 1 & 1 \\
\hline Muslim & $0.67(0.17-2.64)$ & 0.564 & $0.78(0.12-4.85)$ & 0.788 & $1.58(0.34-7.31)$ & 0.547 \\
\hline Christian & $2.3(0.42-13.53)$ & 0.317 & $1.50(0.52-4.29)$ & 0.439 & $3.9(1.26-12.21)$ & 0.019 \\
\hline \multicolumn{7}{|l|}{ Caste } \\
\hline General & 1 & & 1 & & 1 & 1 \\
\hline SC/ST & $2.04(0.25-16.44)$ & 0.493 & $1.22(0.37-3.98)$ & 0.736 & $2.13(0.30-14.93)$ & 0.436 \\
\hline OBC & $1.40(0.11-16.42)$ & 0.782 & $0.17(0.12-2.55)$ & 0.198 & $0.26(0.02-2.40)$ & 0.228 \\
\hline \multicolumn{7}{|c|}{ Monthly family income (₹) } \\
\hline$\leq 10000$ & 1 & & 1 & & 1 & \\
\hline
\end{tabular}




\begin{tabular}{|c|c|c|c|c|c|c|}
\hline \multirow[t]{2}{*}{ Variables } & \multicolumn{2}{|l|}{ Wasting } & \multicolumn{2}{|l|}{ Stunting } & \multicolumn{2}{|l|}{ Underweight } \\
\hline & OR(95\%CI) & p-value & OR(95\%CI) & p-value & OR(95\% CI) & p-value \\
\hline$>10000$ & $0.61(0.41-0.90)$ & 0.016 & $0.50(0.26-0.97)$ & 0.043 & $0.71(0.45-1.12)$ & 0.143 \\
\hline \multicolumn{7}{|l|}{ House type } \\
\hline Kutcha & 1 & & 1 & & 1 & \\
\hline Pucca & $0.83(0.49-1.41)$ & 0.493 & $1.71(0.97-3.01)$ & 0.063 & $1.18(0.74-1.88)$ & 0.458 \\
\hline \multicolumn{7}{|c|}{ Livestock ownership } \\
\hline None & 1 & & 1 & & 1 & \\
\hline 1 or $>$ & 1.37(0.78-2.39) & 0.258 & $2.36(1.25-4.44)$ & 0.009 & $1.39(0.81-2.36)$ & 0.219 \\
\hline \multicolumn{7}{|c|}{ Toilet availability } \\
\hline Yes & 1 & & 1 & & 1 & \\
\hline No & $1.21(0.73-1.99)$ & 0.444 & $1.23(0.81-1.86)$ & 0.308 & $1.47(0.95-2.27)$ & 0.076 \\
\hline \multicolumn{7}{|c|}{ Age at marriage, years } \\
\hline$<18$ & 1 & & 1 & & 1 & \\
\hline$\geq 18$ & $1.05(0.44-2.49)$ & 0.898 & $0.58(0.30-1.12)$ & 0.106 & $0.87(0.40-1.87)$ & 0.724 \\
\hline \multicolumn{7}{|c|}{ Maternal height, cm } \\
\hline$<145$ & 1 & & 1 & & 1 & \\
\hline$\geq 145$ & $0.43(0.21-0.86)$ & 0.020 & $0.69(0.32-1.48)$ & 0.341 & $0.46(0.23-0.95)$ & 0.038 \\
\hline \multicolumn{7}{|c|}{ Maternal nutrition status } \\
\hline $\mathrm{BMI}<18.5$ & 1 & & 1 & & 1 & \\
\hline $\mathrm{BMI} \geq 18.5$ & $0.88(0.53-1.45)$ & 0.613 & $0.63(0.42-0.95)$ & 0.029 & $0.87(0.57-1.34)$ & 0.546 \\
\hline \multicolumn{7}{|c|}{ Place of delivery } \\
\hline Home & 1 & & 1 & & 1 & \\
\hline Institution & $0.77(0.33-1.8)$ & 0.548 & $0.89(0.42-1.86)$ & 0.752 & $0.47(0.19-1.16)$ & 0.101 \\
\hline \multicolumn{7}{|c|}{ Child age, months } \\
\hline $0-6$ & 1 & & 1 & & 1 & \\
\hline $7-23$ & $0.62(0.39-1.00)$ & 0.050 & 5.89(3.03-11.45) & 0.000 & $1.62(0.91-2.86)$ & 0.093 \\
\hline \multicolumn{7}{|l|}{ Child gender } \\
\hline Male & 1 & & 1 & & 1 & \\
\hline Female & $0.70(0.46-1.06)$ & 0.093 & $0.94(0.60-1.45)$ & 0.779 & $0.85(0.52-1.39)$ & 0.527 \\
\hline \multicolumn{7}{|c|}{ Diarrhoea (last 2 weeks) } \\
\hline Yes & 1 & & 1 & & 1 & \\
\hline No & $0.80(0.49-1.30)$ & 0.365 & $0.84(0.48-1.48)$ & 0.556 & $0.62(0.36-1.07)$ & 0.087 \\
\hline
\end{tabular}




\section{DISCUSSION AND CONCLUSIONS}

The prevalence of wasting among children $<2$ years in Narmada was above the critical value of public health significance $(\geq 15 \%)$ [18]. Similarly, the prevalence of underweight and stunting were found to be in a very high category (30-39\%). We identified monthly family income and child age as being associated with both wasting and stunting; households with a low monthly family income had higher odds of having a wasted and stunted child. Younger children had higher odds of wasting, while older children had higher stunting odds. Maternal height was associated with both wasting and underweight; mothers with short stature had higher odds of having a stunted and underweight child. Livestock ownership and low maternal nutritional status were associated with a higher odds of being stunted.

Comparing these 2019 estimates with National Family Health Survey (NFHS-4) data for this district from 2015-16, an improvement was observed in all three estimates- wasting (37.2\% to $32.2 \%$ ), stunting (47.9\% to 34.5\%) and underweight (55.3\% to 39.7\%) [7]. However, these NFHS-4 estimates were for children under 5 years and the present study includes only children under 2 years.

Evidence on growth faltering suggests focusing on the first 1,000 days- from conception to child's second birthday, for setting the foundation of optimum growth, neurodevelopment, and health across the lifespan [1]. Yet, many studies focus on older children. Numerous studies have assessed the prevalence and associated factors of undernutrition among children under five years in high burden countries; however, again very few have focused on younger children [19-22]. Studies from Africa have identified birth weight, child age, diarrhoea (individual level); nutrition status, education level, home delivery, (maternal level); socioeconomic status, number of children, and death of other children (household level) as predictors [23, 24]. Similarly, Asia-based studies found birth weight, child's gender, diarrhoea, immunization status (individual level); height, nutritional status, education level, age at first pregnancy (maternal level); socio-economic status, food security status, and toilet availability (household level) as predictors of undernutrition among under two children [2527]. Unlike these studies, our study did not find household food insecurity, toilet availability, number of children, maternal education level or maternal age at first pregnancy as significant predictors.

In Narmada district, to address the high levels of undernutrition among children that are associated with household livestock keeping and economic conditions, and maternal short stature and nutrition status, a comprehensive approach is needed. Evidence suggests livestock keeping has both positive and negative effects on child undernutrition [28-30]. The positive effects include improved diet quality (access to nutrient-rich animal-source foods) and nutritious food purchase (livestock-derived income), while negative effects include exposure to livestock faeces (leading to diarrhoea or environmental enteric dysfunction). The negative association found in our study suggests a need for educating these communities regarding hygiene practices, especially with respect to livestock.

Maternal stature is an indicator of intergenerational linkages between maternal and child nutrition status and is identified as a strong predictor of child undernutrition in low- and middle-income countries (LMICs) [31]. Pooled analysis from LMICs showed that for every $1 \mathrm{~cm}$ increase in maternal height the risk of stunting was reduced by $4 \%$ and wasting by $1 \%$ $[31,32]$. To break the intergenerational cycle, ensuring adequate adolescent girls' nutrition is critical. Government of India's existing programs, such as public distribution system, and supplementary nutrition have the potential to improve household economic conditions, 
adolescent nutrition, and maternal nutrition status. However, the implementation and coverage of these schemes need to be explored in Narmada District.

This study provided a situational analysis of child undernutrition and information on its predictors for a large nutrition project. The key messages for intervention planning include focusing on adolescent girls, informing households about the existing programs, and education on hygiene practices.

Given the high proportion of infants 0-6 months who were exclusively breastfed, it was surprising that they suffered from more wasting than older infants. We suspect this was likely due to low birth weight, which is very common throughout India. However, we did not obtain data on birth weight.

Findings should be interpreted considering the following limitations. Firstly, as this was a cross-sectional study, one should interpret the associations between exposures and outcomes as causality only with caution. Secondly, child feeding practices could be important determinants but were not explored in this study. Thirdly, we suspect social desirability bias could have affected responses to household food insecurity questions. Despite these limitations, this study also has notable strengths. For anthropometric measurements, we used standard equipment with high precision and trained personnel. We provide district-level estimates of child undernutrition, which can be used as baseline prevalence to evaluate the impact of the POSHAN Abhiyan (the Government of India's strategy to strength existing nutrition interventions/programs) in Narmada district.

\section{Acknowledgements}

We would like to express our heartfelt gratitude to study participants and field investigators.

\section{Funding}

Adani Foundation (AF), a corporate social responsibility Organisation, funded this study. AF did not participate in the design of the study, data collection, analysis, interpretation, or in writing the manuscript.

\section{Availability of data and materials}

The raw data used in this study are available from the corresponding author on request.

\section{Authors' contributions}

RR was involved in all stages (conception and design, data acquisition, analysis and interpretation) and wrote the first draft. AS and RN played major role in data acquisition, AS was also involved in data analysis. DNH was involved in design and analysis. DNH also provided comments on first draft. All authors have approved the final version of this article.

\section{Competing interests}

The authors declare that they have no competing interests. 


\section{REFERENCES}

1. Black RE, Victora CG, Walker SP, Bhutta ZA, Christian P, de Onis M, et al. Maternal and child undernutrition and overweight in low-income and middle-income countries. The Lancet. 2013;382(9890):427-51. doi:10.1016/S0140-6736(13)60937-X

2. Victora CG, Adair L, Fall C, Hallal PC, Martorell R, Richter L, et al. Maternal and child undernutrition: consequences for adult health and human capital. The Lancet. 2008;371(9609):340-57. doi:10.1016/S0140-6736(07)61692-4

3. World Health Organization. Global nutrition targets 2025: Policy brief series. World Health Organization; 2014.

4. World Health Organization. United Nations Decade of Action on Nutrition (20162025)(WHA69. 8). Geneva: World Health Organization. 2016.

5. United Nations Children's Fund, World Health Organization, World Bank. Levels and trends in child malnutrition: key findings of the 2019 Edition of the Joint Child Malnutrition Estimates. Geneva: World Health Organization; 2019.

6. Development Initiatives. 2018 Global Nutrition Report: Shining a light to spur action on nutrition. Bristol, UK; 2018.

7. International Institute for Population Sciences (IIPS), ICF. National Family Health Survey (NFHS-4) 2015-16 India. Mumbai, IIPS; 2017.

8. Swaminathan S, Hemalatha R, Pandey A, Kassebaum NJ, Laxmaiah A, Longvah T, et al. The burden of child and maternal malnutrition and trends in its indicators in the states of India: the Global Burden of Disease Study 1990-2017. The Lancet Child \& Adolescent Health. 2019;3(12):855-70.

9. Nourishing India: National Nutrition Strategy, Government of India [Internet]. Government of India. 2017 [cited 1 September 2019]. Available from: http://niti.gov.in/writereaddata/files/document_publication/Nutrition_Strategy_Booklet.p df.

10. Paul VK, Singh A, Palit S. POSHAN Abhiyaan: Making nutrition a jan andolan. Proceedings of the Indian National Science Academy. 2018;84(4):835-41. doi:https://doi.org/10.16943/ptinsa/2018/49452

11. FAO F. Guidelines for measuring household and individual dietary diversity. Food and Agriculture Organization of the United Nations (FAO) the Food and Nutrition Technical Assistance (FANTA) Project, Rome, Italy. 2007.

12. World Health Organization. Indicators for assessing infant and young child feeding practices: part 2: measurement. 2010.

13. Coates J, Swindale A, Bilinsky P. Household Food Insecurity Access Scale (HFIAS) for measurement of food access: indicator guide: version 3. 2007.

doi:https://www.fantaproject.org/monitoring-and-evaluation/household-food-insecurityaccess-scale-hfias

14. Cashin K, Oot L. Guide to Anthropometry: A Practical Tool for Program Planners, Managers, and Implementers. Food Nutr Tech Assist III Proj (FANTA)/FHI. 2018;360:93-115.

15. World Health Organization. WHO Child Growth Standards: methods and development, Length/height-for-age, weight-for-age, weight-for-length, weight-for-height and body mass index-for-age. Geneva; 2006.

16. Leroy JL. zscore06: Stata command for the calculation of anthropometric z-scores using the 2006 WHO child growth standards. International Food Policy Research Institute, Washington DC2011 [Available from: http://fmwww.bc.edu/repec/bocode/z/zscore06.html. 
17. Crowe S, Seal A, Grijalva-Eternod C, Kerac M. Effect of nutrition survey 'cleaning criteria' on estimates of malnutrition prevalence and disease burden: secondary data analysis. PeerJ. 2014;2:e380. doi:10.7717/peerj.380

18. World Health Organization. NLIS country profile indicators interpretation guide. Geneva (Switzerland): World Health Organization. 2010.

19. Wete AT, Zerfu TA, Anbese AT. Magnitude and associated factors of wasting among under five orphans in Dilla town, southern Ethiopia: 2018: a cross-sectional study. BMC Nutrition. 2019;5(1):33.

20. Adhikari RP, Shrestha ML, Acharya A, Upadhaya N. Determinants of stunting among children aged 0-59 months in Nepal: findings from Nepal Demographic and health Survey, 2006, 2011, and 2016. BMC Nutrition. 2019;5(1):37. doi:https://doi.org/10.1186/s40795-019-0300-0

21. Ma'alin A, Birhanu D, Melaku S, Tolossa D, Mohammed Y, Gebremicheal K. Magnitude and factors associated with malnutrition in children 6-59 months of age in Shinille Woreda, Ethiopian Somali regional state: a cross-sectional study. BMC Nutrition. 2016;2(1):44.

22. Meshram I, Rao K, Reddy C, Sharad K, Sreerama K, Hari K. Prevalence of under nutrition and its predictors among under 5 year children in Surat region, Gujarat, India. Journal of Clinical Nutrition \& Dietetics. 2016;2:1-12.

23. Nkurunziza S, Meessen B, Korachais C. Determinants of stunting and severe stunting among Burundian children aged 6-23 months: evidence from a national cross-sectional household survey, 2014. BMC Pediatrics. 2017;17(1):176.

24. Humbwavali JB, Giugliani C, Nunes LN, Dalcastagnê SV, Duncan BB. Malnutrition and its associated factors: a cross-sectional study with children under 2 years in a suburban area in Angola. BMC Public Health. 2019;19(1):220.

25. Ahmed T, Roy S, Alam N, Hossain MI. Determinants of undernutrition in children under 2 years of age from rural Bangladesh. Indian Pediatrics. 2012;49(10):821-4. doi:10.1007/s13312-012-0187-2

26. Tariq J, Sajjad A, Zakar R, Zakar MZ, Fischer F. Factors associated with undernutrition in children under the age of two years: secondary data analysis based on the Pakistan demographic and health survey 2012-2013. Nutrients. 2018;10(6):676.

27. Mistry SK, Hossain MB, Khanam F, Akter F, Parvez M, Yunus FM, et al. Individual-, maternal-and household-level factors associated with stunting among children aged 0-23 months in Bangladesh. Public Health Nutrition. 2019;22(1):85-94.

28. De Bruyn J, Thomson PC, Darnton-Hill I, Bagnol B, Maulaga W, Alders RG. Does village chicken-keeping contribute to young children's diets and growth? A longitudinal observational study in rural Tanzania. Nutrients. 2018;10(11):1799.

29. Hetherington JB, Wiethoelter AK, Negin J, Mor SM. Livestock ownership, animal source foods and child nutritional outcomes in seven rural village clusters in SubSaharan Africa. Agriculture \& Food Security. 2017;6(1):9.

30. Leroy JL, Frongillo EA. Can interventions to promote animal production ameliorate undernutrition? the Journal of Nutrition. 2007;137(10):2311-6.

31. Khatun W, Rasheed S, Alam A, Huda TM, Dibley MJ. Assessing the Intergenerational Linkage between Short Maternal Stature and Under-Five Stunting and Wasting in Bangladesh. Nutrients. 2019;11(8):1818.

32. Subramanian S, Ackerson LK, Smith GD, John NA. Association of maternal height with child mortality, anthropometric failure, and anemia in India. JAMA. 2009;301(16):1691701. 